\title{
Cathodoluminescence Detectors Suitable for Easy and Fast Analysis in Geosciences
}

\author{
Jaroslav Jiruše ${ }^{1}$, Jolana Kološová ${ }^{1}$, Jaromír Leichmann ${ }^{2}$ \\ 1. TESCAN, a.s., Brno, Czech Republic \\ 2. Faculty of Science, Masaryk University, Brno, Czech Republic
}

Cathodoluminescence (CL) - light emission produced by electron beam - has become a powerful method in geosciences. Typical application examples are provenance analysis of sedimentary rocks, analysis of structure and crystal chemistry of solids, and reconstruction of processes of mineral formation and alteration. With the development of scanning electron microscopy (SEM), scanned CL is gaining in popularity $[1,2,3]$. In comparison with optical CL microscopes, SEM-based CL imaging achieves much better resolution and it can be combined with other techniques like SE, BSE, EDX, EBIC and others.

TESCAN has developed both panchromatic and color types of CL detectors. They are fully integrated into the SEMs software and there is no need for external scanning. This is a huge advantage compared to existing commercial systems. The panchromatic detector has maximal spectral range from $185 \mathrm{~nm}$ to $850 \mathrm{~nm}$. A recently developed color CL detector has a spectral range of $350 \mathrm{~nm}$ to $850 \mathrm{~nm}$. The RGB parts of the signal are acquired simultaneously and are electronically composed into a live color image of the scanned area, see Figure 1. As can be seen by comparison with hot-cathode CL, the scanned CL has much better resolution and individual zones are more distinct. All image processing is integrated in the SEM software, which is capable of processing color pictures.

Both of these CL detectors are easily adjustable and can be retracted form their working position if not in use. The SEM software enables simultaneous acquisition of up to four different signals, for example CL with SE or BSE etc. To facilitate the analysis of large samples, sophisticated software modules are available (the user can search for areas of interest on the sample and store their position in memory for repeated analysis, or acquire individual images and compose them into large panoramic pictures).

Thanks to their original design, new CL detectors are not overly sensitive to sample height or position, see Figure 2. The field of view (FOV) is extremely large using TESCAN Wide Field Optics ${ }^{\mathrm{TM}}$ technology. Up to $35 \mathrm{~mm}$ (width) FOV can be imaged with a single beam scan - no sample/stage scanning is needed and thus simultaneous CL acquisition with other SEM signals is easy. This is extremely valuable compared to conventional $C L$ detectors with a field of view as small as $10 \mu \mathrm{m}$. Such large surveillance snapshots are ideal for quick orientation on the sample. Moreover, unpolished samples - even samples that are not flat - can be imaged in CL with uniform signal collection efficiency over the full FOV. Excellent sensitivity of new detectors maintains high signal to noise ratio even at low accelerating voltages, which minimizes sample radiation damage and, thanks to the smaller electronspecimen interaction volume, enables the acquisition of images with the outstanding resolution. And finally, sensitive samples can be left uncoated and analyzed in the low vacuum mode. All these features make new CL detectors ideal tools for an easy and fast analysis in geosciences [4]. 


\section{References:}

[1] DK Richter et al, Mineralogy and Petrology 79 (2003) p. 127.

[2] J Götze, U Kempe, Mineralogical Magazine 72 (2008) p. 909.

[3] S Boggs, JD Kinsley, "Application of Cathodoluminescence Imaging to the Study of Sedimentary Rocks", (Cambridge University Press, New York).

[4] The authors acknowledge funding from the European Union FP7 Programme under grant agreement No. 280566, project UnivSEM.
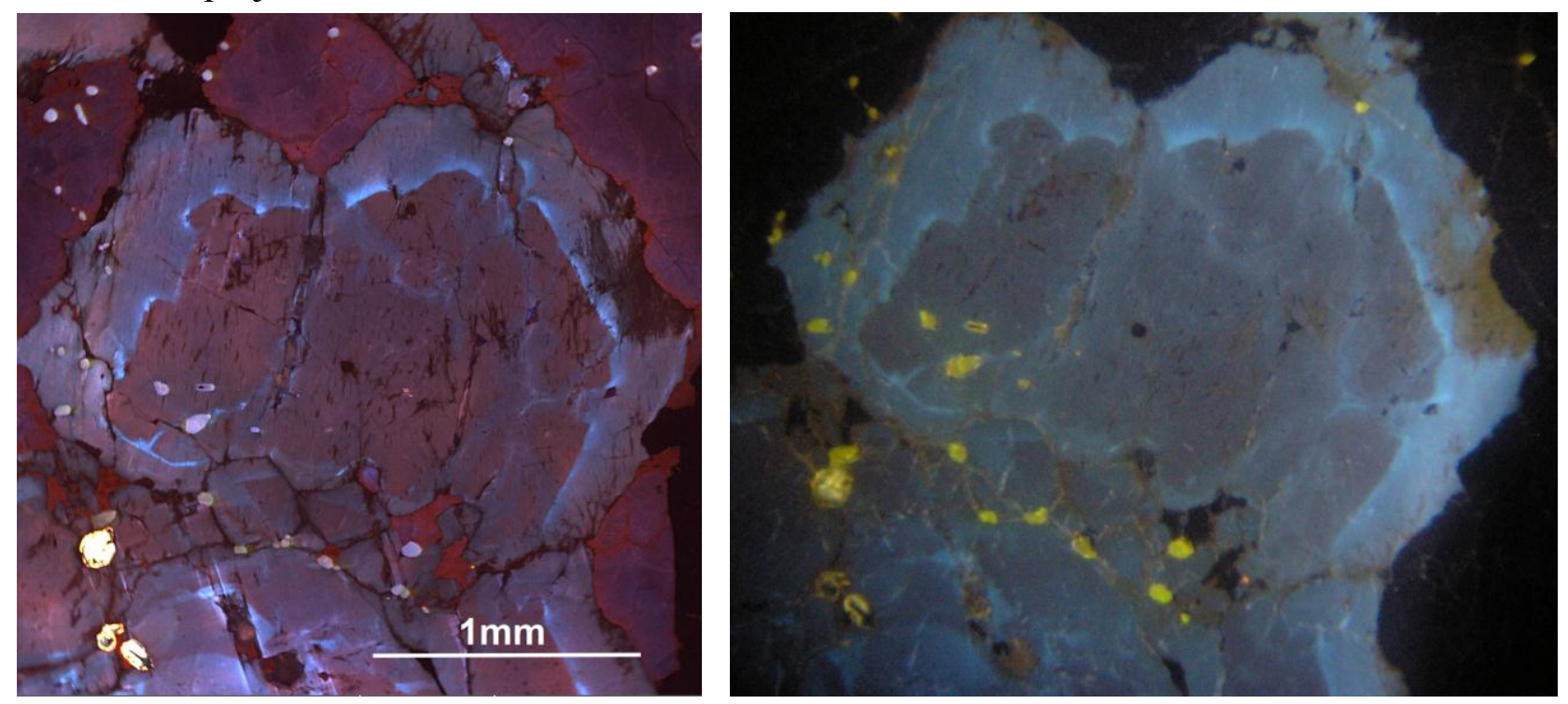

Figure 1. Polished feldspar sample, color scanned CL image (left) in comparison with hot-cathode CL image of the same area (apatite grains are strongly luminescent). Resulting colors are a little shifted, but the scanned image has better resolution and individual zones are more distinct.
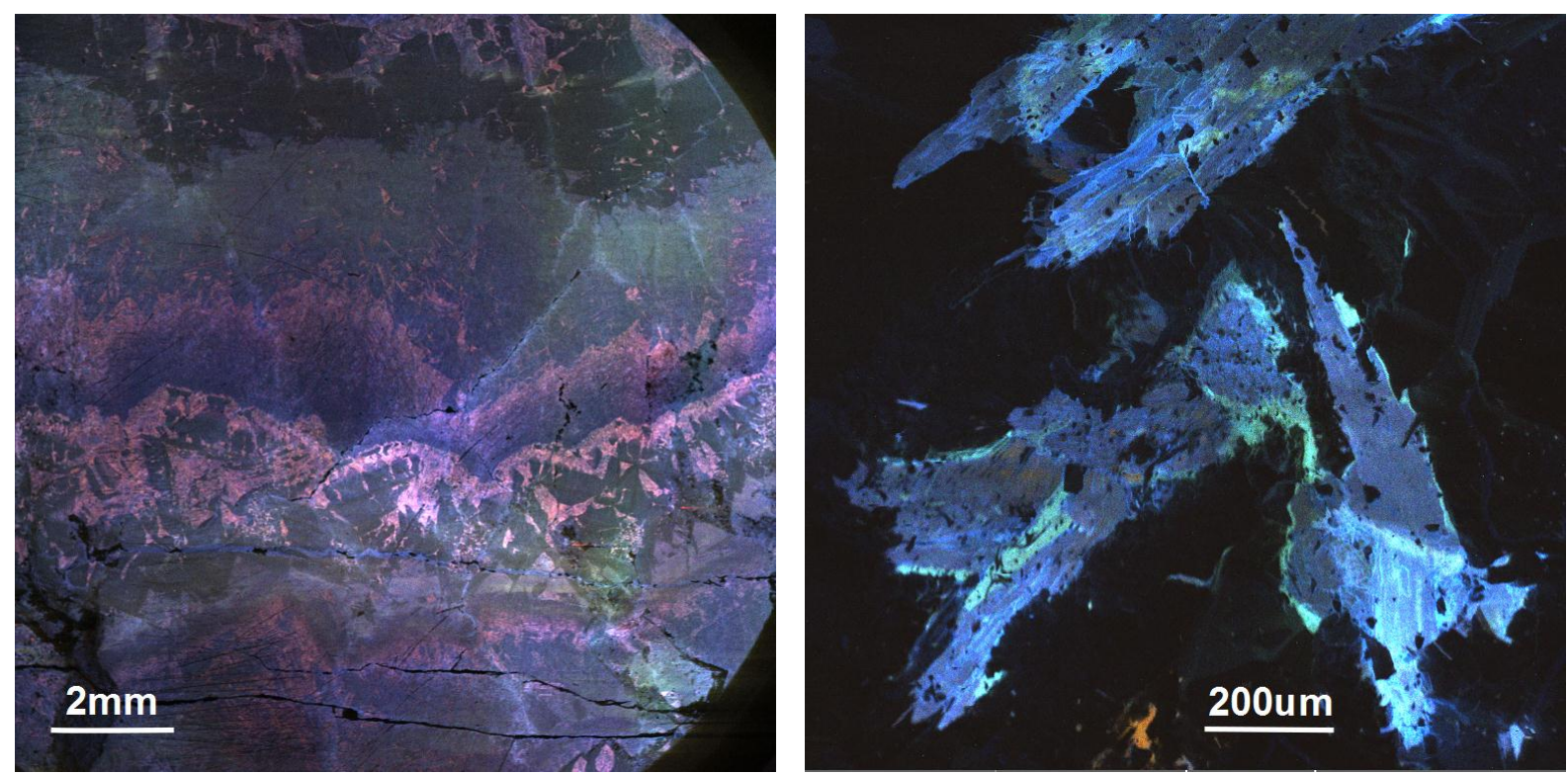

Figure 2. A large single scan (no sample scanning) of a fluorite sample (left) and an image of an unpolished rock chip with brightly luminescent karpatite crystals (right). Detection efficiency does not change dramatically with primary beam position in spite of $13 \mathrm{~mm}$ field of view and $3 \mathrm{~mm}$ difference in sample height topography. 